\title{
Non-inflammatory and non-neoplastic soft tissue lesions of the tympanic cavity
}

\author{
Gülen Burakgazi' $^{1}$ (D), Hanifi Bayaroğullari² (D) \\ ${ }^{1}$ Department of Radiology, School of Medicine, Recep Tayyip Erdoğan University, Rize, Turkey \\ ${ }^{2}$ Department of Radiology, School of Medicine, Mustafa Kemal University, Hatay, Turkey
}

\begin{abstract}
Objectives: Tympanic cavity (TC) is an anatomically challenging region for the diagnosis of lesions located inside it. Radiological diagnosis and demonstration of the anatomic localization of sporadic lesions such as vascular malformations and meningocele are essential for the prevention of complications that may occur during operation and recurrent unnecessary interventions. The aim of the present study was to demonstrate the radiological appearance of the vascular variations and the post-traumatic soft tissue lesions which are rarely encountered in TC.
\end{abstract}

Methods: A total of 3525 temporal multi-slice computed tomography (MSCT) images were evaluated between 2010-2018. Twelve cases with non-inflamatory and non-neoplastic soft tissue lesions in TC were detected and 2 with meningocele were included in the study.

Results: Of the 12 cases, 6 were males and 6 were females. The mean age of the patients was 26.16 years. Four cases had an aberrant internal carotid artery (ICA), being bilateral in 2 cases and right sided in the remaining 2 cases. Six cases had dehiscent mega jugular bulb (DMJB); and 2 cases had post-traumatic meningocele.

Conclusion: Vascular malformations and meningoceles are rarely observed in TC. They may present nonspecific clinical signs and symptoms; however, they should be evaluated with MSCT and MRI before the surgical interventions regarding the middle ear to prevent possible complications.

Keywords: meningocele; radiologic finding; tympanic cavity; vascular malformation

Anatomy 2020;14(3):157-164 (2020 Turkish Society of Anatomy and Clinical Anatomy (TSACA)

\section{Introduction}

Temporal bone has a complicated structure since it contains two of the cranial nerves (facial and vestibulocochlear nerves), blood vessels such as meningeal arteries and internal carotid artery (ICA) and elements of vestibule-auditory system. The tympanic cavity (TC) is a rectangular cavity with 6 walls located within the petrosal part of the temporal bone. The lateral wall consists of the tympanic membrane and epitympanic recess, the medial wall is formed by the lateral wall of the inner ear featuring the oval and round windows. The roof (tegmental wall) separates the TC from the dura mater of the middle cranial fossa. The floor (jugular wall) forms the base of the hypotympanum and provides separation from the jugular bulb. The anterior wall is a thin bone plaque with an opening for eustachian tube and tensor tympani muscle. This wall sep- arates ICA from TC. The posterior wall separates mastoid cells from TC. TC is divided into three parts by taking the tympanic membrane as the key point. The upper part of the tympanic membrane is described as epitympanum, the lower part as hypotympanum and the tympanic membrane level as mesotympanum. A bone chain consisting of manubrium, incus, and stapes in mesotympanum allows connection between the external ear and inner ear. ${ }^{[1,2]}$ The complicated structure, vital neurovascular relationships and relative small area of TC makes the clinical and radiological evaluation of lesions in this region challenging. The soft tissue masses, located in TC could be evaluated by otoscopic examination and all the details in this area could be shown more clearly by radiological examinations such as multislice computed tomography (MSCT) and magnetic resonance imaging (MRI). ${ }^{[3}$ 
In this study, we aimed to present the vascular variations and the radiological appearance of the post-traumatic soft tissue lesions that are rarely encountered in TC.

\section{Materials and Methods}

Patients who referred to Radiology Department after being evaluated in Mustafa Kemal University hospital due to hearing problems, tinnitus, dizziness, trauma and posttraumatic control between 2010-2018 were retrospectively reviewed. Totally, radiological images of 3525 patients were evaluated for presence of any vascular variations and post-traumatic soft tissue lesions in the TC. Any infectious or neoplastic pathologies causing soft tissue appearance were excluded. Non-inflammatory and non-neoplastic soft tissue lesions were detected in TC of 12 patients who underwent temporal MSCT (Toshiba Aquillon 64 MDCT, Tustin, CA, USA). The evaluation of the cases with MSCT was done by the Basic Vitrea 2 (version 4.0) workstation. In addition to this, four of the patients underwent temporal MRI (1.5 Tesla Philips MRI, Achieva, Best, the Netherlands) for further differential diagnosis of soft tissue lesions. The diagnosis was made with the pathological contrast enhancement in contrast-enhanced MSCT and contrast-enhanced MRI. In addition, CT angiography and MR angiography examinations were made to show vascular structures.

\section{Results}

The mean age of the patients with non-neoplastic and non-inflammatory soft tissue lesions was 26.16 (range: $17-45$ ) years. Six of them were male, and six were female. In 5 of the patients, hearing related findings (total hearing loss in three cases, conductive type hearing loss in two) were the main symptoms. Tinnitus and ear pain was present in 3, non-suppurative otitis media in 3, suppurative otitis media and ear pain in 1 . Two cases had a history of trauma, and total hearing loss and one patient had peripheral facial paralysis. The age, gender, clinical and radiological findings of these 12 patients were summarized in Table $\mathbf{1}$.

Four patients had aberrant internal carotid artery (ICA) in TC (Figure 1). Bilateral ICA was detected in 2 of these cases, and right aberrant ICA was found in the remaining 2 cases (Figure 2). One of the cases with aberrant bilateral ICA had total hearing loss, two cases had otitis media, and one case had tinnitus and ear pain.

A "mega" jugular bulb with dehiscence extending to the TC was present in 6 of the cases. In one case, the jugular

Table 1

Age, gender, clinical and radiological findings of 12 cases.

\begin{tabular}{|c|c|c|c|c|}
\hline No & Age & Gender & Clinical findings & Radiological diagnosis \\
\hline 1 & 22 & $\mathrm{~F}$ & Bilateral total hearing loss & Bilateral aberrant ICA \\
\hline 2 & 17 & $\mathrm{M}$ & Suppurative otitis media, ear pain & R- aberrant ICA \\
\hline 3 & 34 & $\mathrm{~F}$ & Ear pain and tinnitus & R- aberrant ICA \\
\hline 4 & 35 & $\mathrm{~F}$ & Non-suppurative otitis media & $\begin{array}{l}\text { Bilateral aberrant ICA, Close relationship } \\
\text { with bone chain on the right }\end{array}$ \\
\hline 5 & 21 & $M$ & $\begin{array}{l}\text { Right conductive hearing loss, } \\
\text { suppurative otitis media }\end{array}$ & R- DMJB \\
\hline 6 & 18 & $\mathrm{~F}$ & Non-suppurative otitis media & R-DMJB \\
\hline 7 & 22 & K & Non-suppurative otitis media & L- DMJB \\
\hline 8 & 42 & M & Right conduction-type hearing loss & R- DMJB \\
\hline 9 & 45 & M & Tinnitus, ear pain and fullness & R- DMJB \\
\hline 10 & 18 & $\mathrm{~F}$ & Tinnitus & R-DMJB \\
\hline 11 & 22 & M & $\begin{array}{l}\text { Traffic accident } 2 \text { years ago, hearing loss } \\
\text { in the right ear, and facial paralysis }\end{array}$ & $\begin{array}{l}\text { The transfer fracture line in petrous bone } \\
\text { that starting from apical level of ICA and } \\
\text { passing in front of the cochlea, the meningocele } \\
\text { sac extending along this line to the tympanic cavity. }\end{array}$ \\
\hline 12 & 18 & $\mathrm{~F}$ & $\begin{array}{l}\text { Falling from height10 years ago, } \\
\text { total hearing loss in the left ear }\end{array}$ & $\begin{array}{c}\text { Transverse fracture in the left petrous bone, jugular } \\
\text { foramen walls. Cochlear labyrinthitis ossificans, } \\
\text { dislocation in the bone chain, pseudopenningocele } \\
\text { extending to the tympanic cavity at the oval } \\
\text { window level. }\end{array}$ \\
\hline
\end{tabular}

DMJB: dehiscent mega jugular bulb; F: female; ICA: internal carotid artery; L: left; M: male; R: right. 

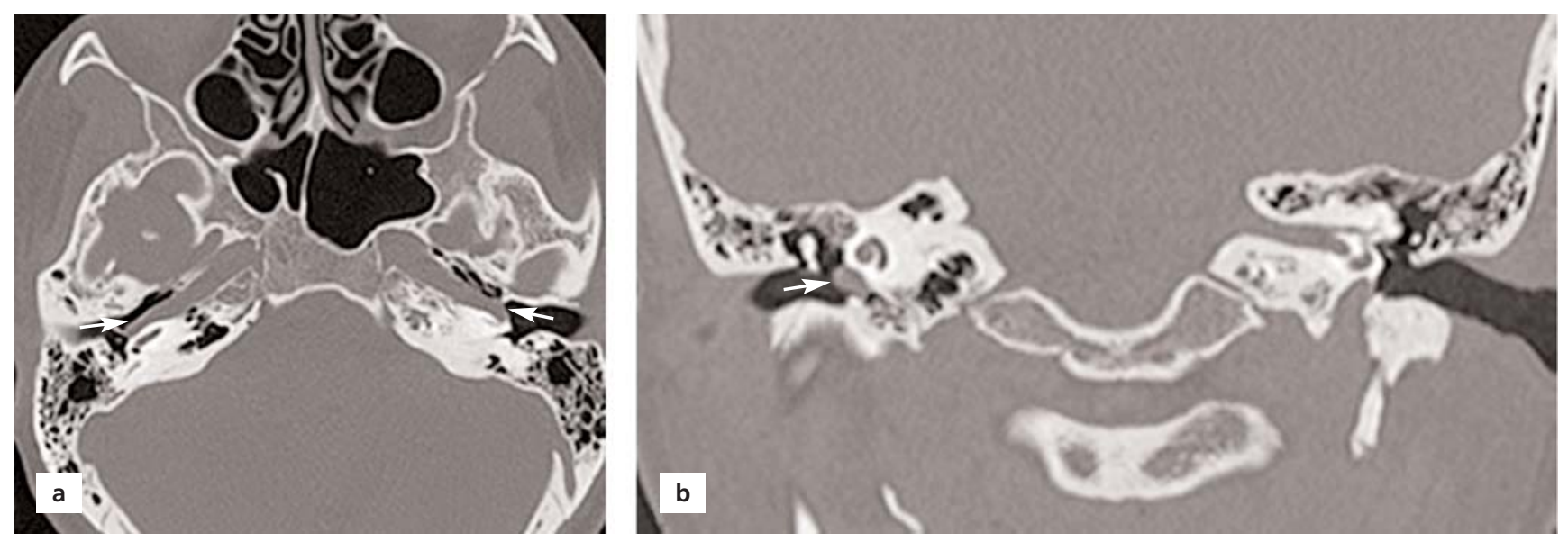

Figure 1. (a) Axial and (b) coronal MSCT sections of a 17-year-old male patient, aberrant ICA in contact with tympanic membrane in the right tympanic cavity (white arrows).

bulb contacted the lower edge of the tympanic membrane (Figure 3). Two patients with dehiscent mega jugular bulb (DMJB) had right conductive hearing loss, one patient had tinnitus and aural fullness, while the other three patients were asymptomatic. The patients with otitis and ear pain had a DMJB that was detected in temporal MSCT. In six cases, temporal MSCT examination showed that jugular bulb caused dehiscence in the bone (Figure 4).

The temporal MSCT obtained from 2 patients with post-traumatic total hearing loss revealed old transverse
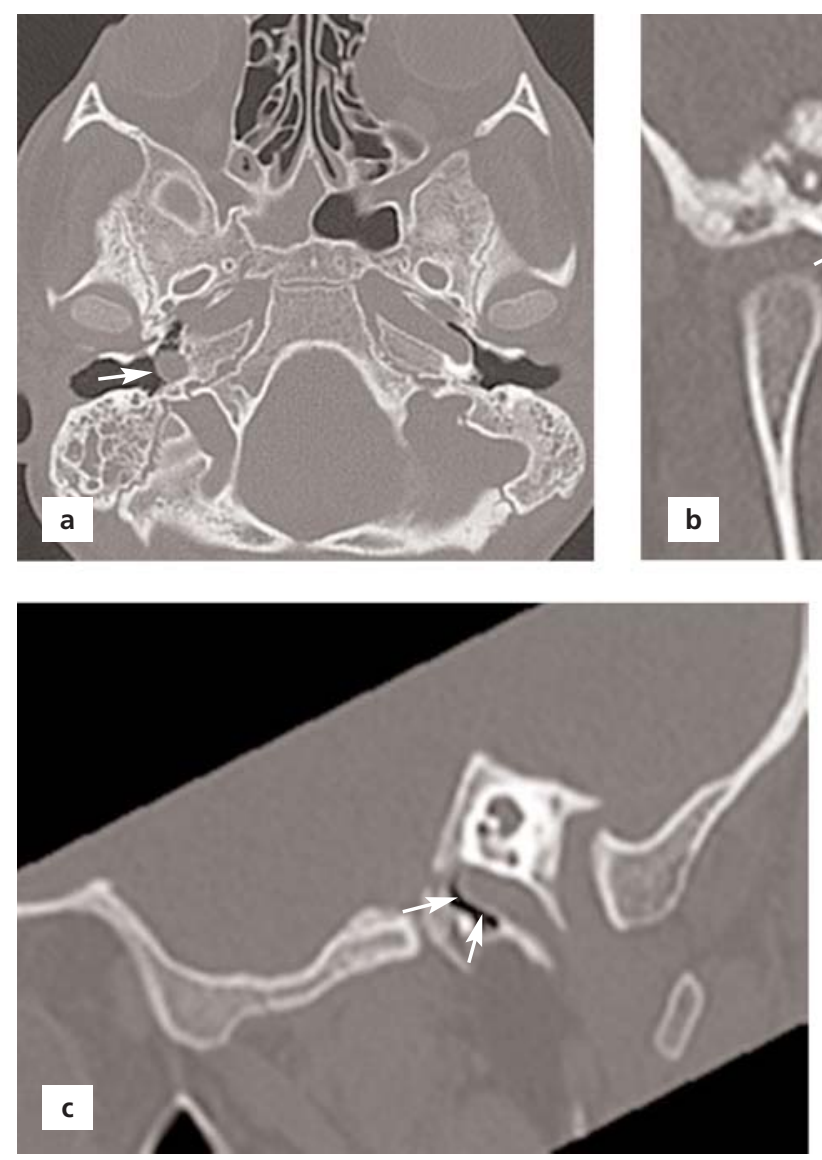

Figure 2. (a) Axial (b) coronal and (c) sagittal MSCT sections of a 35-yearold female patient, bilateral aberrant ICA on the right is more prominent and in contact with the tympanic ossicles (white arrows). 

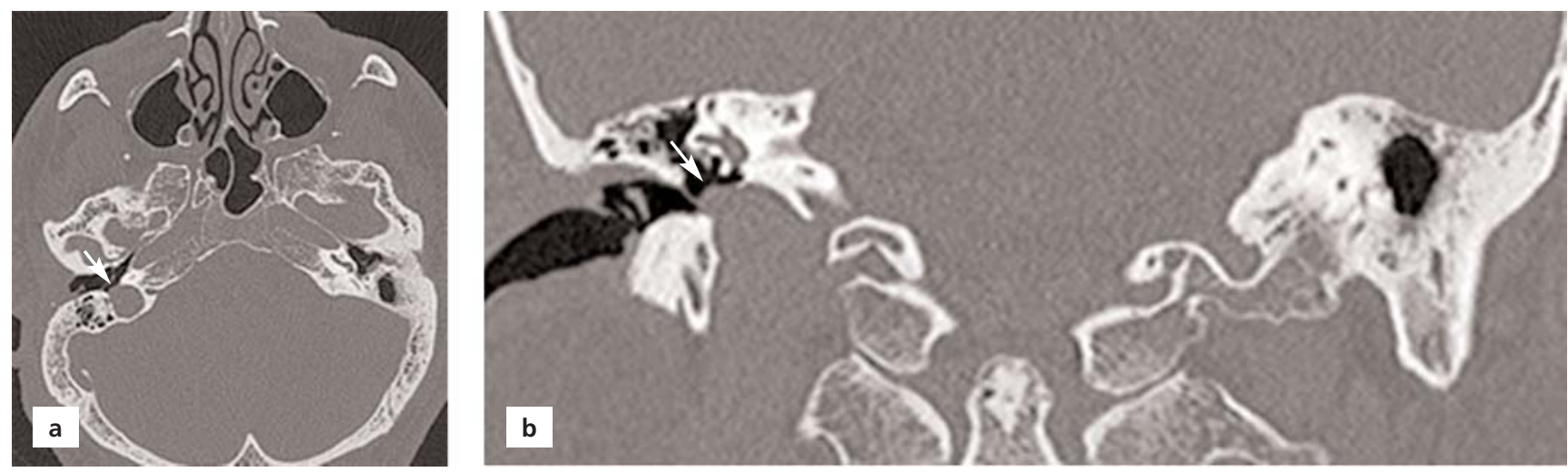

Figure 3. (a) Axial and (b) coronal MSCT sections of a 42-year-old male patient, mega-jugular bulbus which caused dehiscence on the base of tympanic cavity, extending to the tympanic cavity and touching the tympanic membrane (white arrows).

fracture lines in the right petrous bone including the otic capsule. The first case was a 22 -year-old male with a previous motor vehicle accident happened 2 years ago. The patient had total hearing loss and peripheral facial paralysis in the right ear after the trauma. The temporal MSCT revealed a fracture line starting from the apical level of IAC, passing close to the cochlea and reaching to TC by passing through the labyrinthine part of the facial nerve. In addition to this, a meningocele was detected by MSCT and MRI in the same patient (Figure 5). The second case with meningocele was an 18-year-old woman. She had a total hearing loss in her left ear after falling from a height 8 years ago. A transverse fracture in the petrous bone was detected by MSCT and MRI just in front of the cochlea, which passed from the proximal part of the tympanic portion of the facial nerve. The fracture caused dislocation of the stapes separating it from the oval window. A meningocele sac extending from the left oval window to TC was also detected. In this case, labyrinthitis ossificans developed in cochlea (Figure 6).

\section{Discussion}

Any inflammatory, neoplastic and/or vascular soft tissue or bony lesions may be encountered in TC. The soft tissue lesions of TC include cholesteatoma, paraganglioma, aberrant carotid artery, schwannoma, meningocele, and high mega jugular bulb. ${ }^{[1,4]}$ Hearing loss, ear pain and tinnitus are among common symptoms of these patients. Soft tissue lesions in TC are diagnosed by otoscopic or radiologic examinations. The change in the color of the tympanic membrane can easily be detected by otoscopic examination which provides essential information for the several lesions. A red tympanic membrane is a sign for aberrant ICA and glomus tumor in TC; a blue tympanic
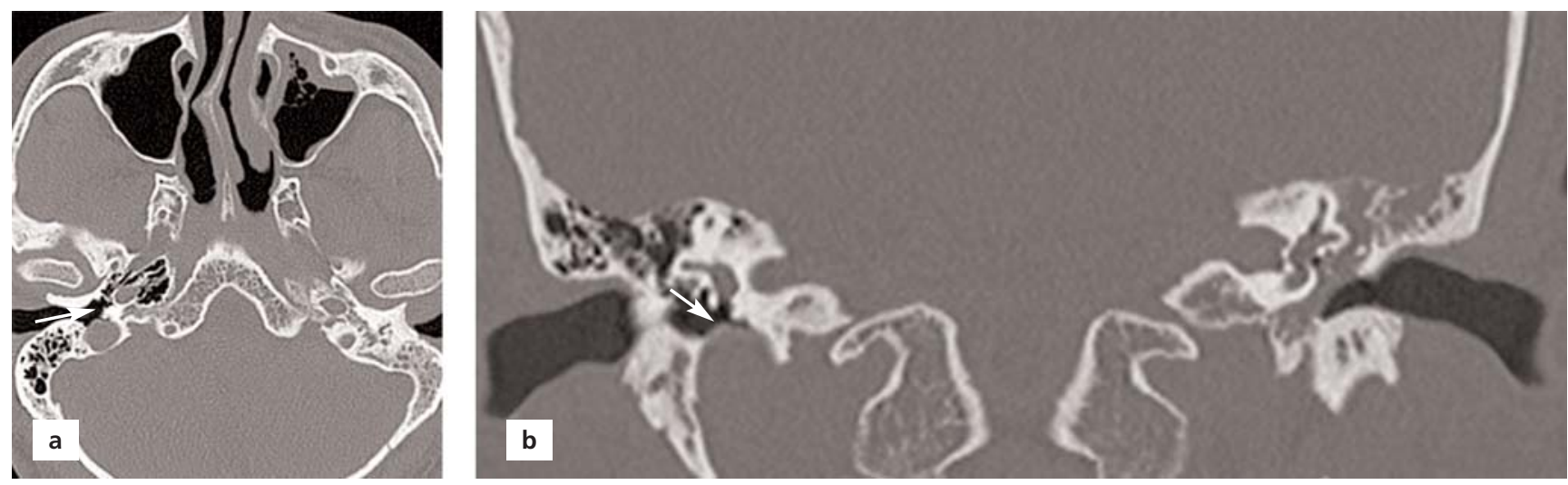

Figure 4. (a) Axial and (b) coronal MSCT sections of a 45-year-old male patient, right mega-jugular bulbus which caused dehiscence on the base of tympanic cavity and extending to the tympanic cavity (white arrows). 

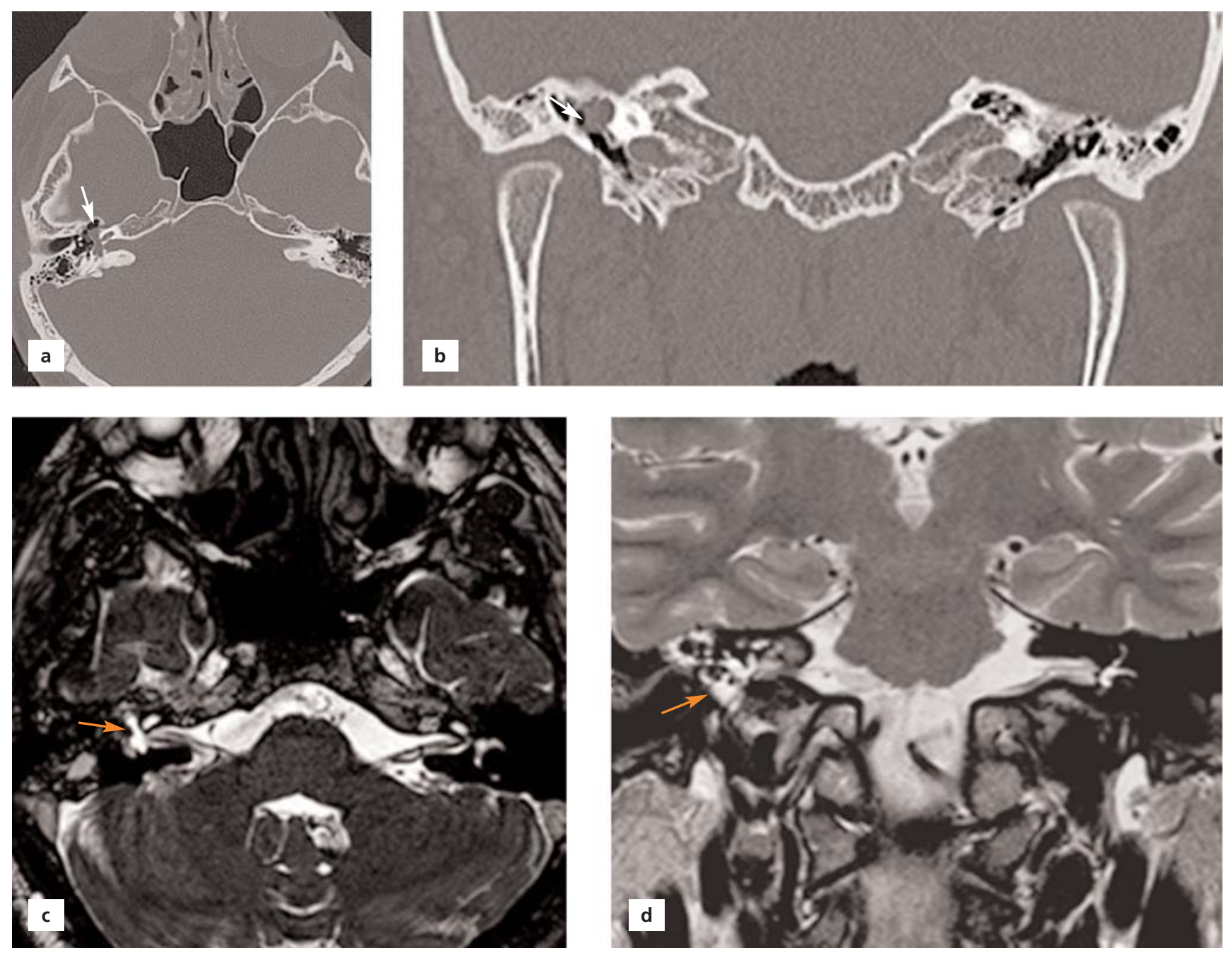

Figure 5. (a) Axial and (b) coronal MSCT sections of a 22-year-old male patient, meningocele pouch is located at the fissure line which crossed internal acoustic canal, labyrinthine part of the facial nerve and reached tympanic cavity (white arrows); (c) axial and (d) coronal T2-weighted MR images showing meningocele pouch in tympanic cavity (orange arrows).

membrane for DMJB, meningocele, meningoencephalocele and cholesterol granuloma; and a white tympanic membrane for the primary tympanic cavity tumors such as congenital cholesteatoma and schwannoma. However, making a differential diagnosis among these lesions is difficult by otoscopic examination thus multiplanar imaging techniques such as MSCT and MRI are gold standard for evaluating TC. It is possible to obtain thin sections with MSCT making it easy to interpret the structures located within the TC in details. MRI is used to evaluate soft tissue lesions and their relationships, while MRI angiography provides useful information for vascular structures. ${ }^{[1,5-7]}$

An aberrant ICA and a DHJB are not so common. Typically, ICA is located in the carotid canal of the petrous part of the temporal bone and is adjacent to the anterior wall of TC. The aberrant ICA in TC is known to occur by the combination of inferior tympanic artery with the carotico-tympanic artery. In such a case, the ICA extends more laterally into the TC. The diagnosis is difficult with the clinical examination because the symptoms are nonspecific. Diagnosis is made with MSCT, which could take thin sections. Vascular pathologies such as glomus tumor, cholesterol granuloma, DMJB, hemangioma, aneurysm of the petrosal segment of the ICA should be considered in the differential diagnosis. ${ }^{[6,8,9]}$ Bilateral aberrant ICA was observed in 2 out of 4 aberrant ICA cases, and 2 cases had an unilateral aberrant ICA on the right TC.

The jugular bulb is the junctional region of the sigmoid sinus and the internal jugular vein and is separated 

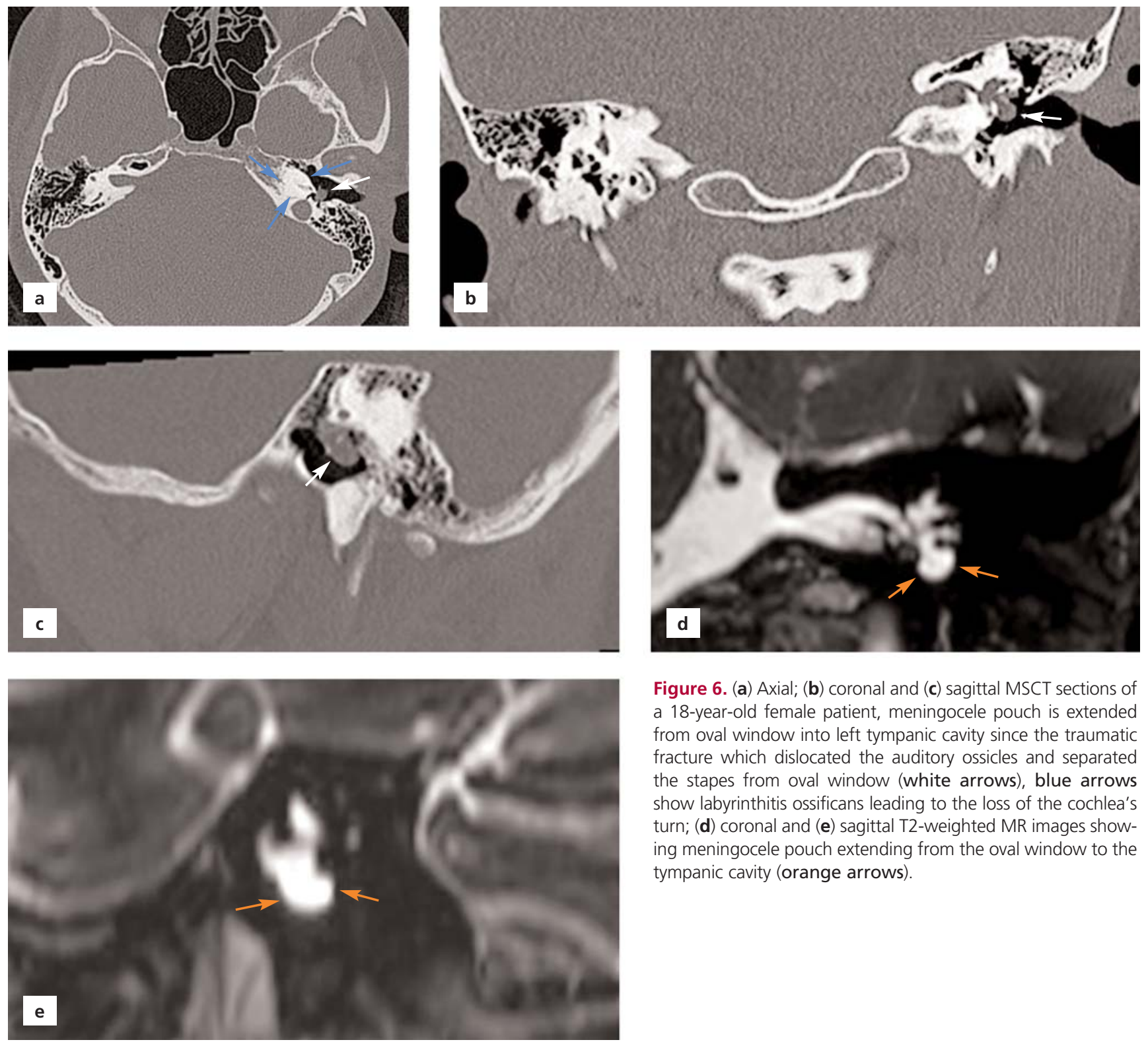

Figure 6. (a) Axial; (b) coronal and (c) sagittal MSCT sections of a 18-year-old female patient, meningocele pouch is extended from oval window into left tympanic cavity since the traumatic fracture which dislocated the auditory ossicles and separated the stapes from oval window (white arrows), blue arrows show labyrinthitis ossificans leading to the loss of the cochlea's turn; (d) coronal and (e) sagittal T2-weighted MR images showing meningocele pouch extending from the oval window to the tympanic cavity (orange arrows).

from the compact bone (hypotympanum) that forms the base of the middle ear. If the upper edge of the jugular bulb extends over the superior part of the fibrocartilaginous ring of the tympanic membrane or above the basal fold of the cochlea, it is considered as high jugular bulb; whereas if the transverse diameter of the bulb is increased it is considered as mega jugular bulb. Localization and diameter of the jugular vein is subject to a wide range of variations. The high and mega jugular bulb could extend into the middle ear cavity and could be with or without a thin bony septum. The incidence of DMJB is reported to be $0.5-1.7 \%$. It is more frequently observed on the right side. It may be asymptomatic or may cause tinnitus and conductive hearing loss. ${ }^{[10,11]}$ Conductive hearing loss was present in 2 of our cases with DMJB. The other 4 cases had otitis, ear pain, and tinnitus in their physical examinations.

Temporal bone fractures occur after severe highenergy-blunt head trauma and develop most frequently after the motor vehicle accidents. Falling from a height, industrial injuries are among the other reasons. They constitute $30-70 \%$ of the calvarial fractures. Temporal bone fractures are classified according to their position on the petrous bone axis as longitudinal, transverse and 
mixed type. In a new classification system, it is classified as including the otic capsule (10\%) or not (90\%), which could better determine the occurrence and prognosis of posttraumatic complications such as hearing loss. Hearing loss is the most common clinical finding, and clinical findings vary according to the type of fracture in the acute and chronic period. Although longitudinal fractures are more common, transverse fractures are more dangerous, and their complications are more frequent. Complications are seen more frequently in the fractures related with the otic capsule. Dislocation in the middle ear ossicles and conduction-type hearing loss, tympanic membrane rupture, hemotympanum and facial nerve paralysis are more frequently observed in longitudinal fractures, whereas sensorineural hearing loss, facial nerve paralysis, and labyrinth fistula are observed in transverse type fractures. In the mixed type fracture, a mixture of these findings may be observed. Labyrinthitis ossificans, which have been reported far less frequently after trauma, could also be seen secondary to the posttraumatic repair process. Post-traumatic vascular injuries, other cranial nerve injuries and meningitis may develop as well. Meningocele/meningoencephalocele are observed rarely. Meningocele formation after trauma is reported in $11 \%$ of the cases and after surgical interventions in $59 \% \cdot{ }^{[7,12-15]}$ One of the meningocele cases in our study had total hearing loss and peripheral nerve paralysis in the right ear. The other case with meningocele was characterized with post-traumatic labyrinthitis ossificans. To our knowledge, no similar meningocele cases were previously reported possessing such a fracture line. Labyrinthitis ossificans observed in the second case is also very rare after trauma.

The limitation of our study was inclusion of only retrospective cases. However, the number of the images evaluated are considerably high to draw a conclusion.

\section{Conclusion}

If the vascular variations in TC such as aberrant ICA and DMJB are not recognized before the operations such as tympanoplasty or cochlear implant placement, there may be severe neurovascular complications. In meningocele, inappropriate and unnecessary interventions would cause perilymphatic fistula and leakage of cerebrospinal fluid, and therefore may lead to severe complications. Consequently, a detailed radiological evaluation with multiplanar MSCT and MRI will be useful before applying any interventions in the middle ear.

\section{Conflict of Interest}

No conflict of interest was declared by the authors.

\section{Author Contributions}

The authors equally contributed to concept, design, data processing, literature reviewing, data analysis and interpretation, and writing manuscript.

\section{Ethics Approval}

The study was approved by Mustafa Kemal University Clinical Research Ethics Committee (Ethics No: 2019/81). The study was also carried out in accordance with the Helsinki Declaration of Principles.

\section{Funding}

The authors declare that this study has not received any financial support.

\section{References}

1. Juliano AF, Ginat DT, Moonis G. Imaging review of the temporal bone: part I. Anatomy and inflammatory and neoplastic processes. Radiology 2013;269:17-33.

2. Luers JC, Hüttenbrink KB. Surgical anatomy and pathology of the middle ear. J Anat 2016;228:338-53.

3. Wang CH, Shi ZP, Liu DW, Wang HW, Huang BR, Chen HC. High computed tomographic correlations between carotid canal dehiscence and high jugular bulb in the middle ear. Audiol Neurootol 2011;16:106-12.

4. Anbarasu A, Chandrasekaran K, Balakrishnan S. Soft tissue attenuation in middle ear on HRCT: pictorial review. Indian J Radiol Imaging 2012;22:298-304.

5. Trojanowska A, Drop A, Trojanowski P, Rosinska-Bogusiewicz K, Klatka J, Bobek-Billewicz B. External and middle ear diseases: radiological diagnosis based on clinical signs and symptoms. Insights Imaging 2012;3:33-48.

6. Yanmaz R, Okuyucu Ş, Burakgazi G, Bayaroğullari H. Aberrant internal carotid artery in the tympanic cavity. J Craniofac Surg 2016; 27:2001-3.

7. Zayas JO, Feliciano YZ, Hadley CR, Gomez AA, Vidal JA. Temporal bone trauma and the role of multidetector CT in the emergency department. Radiographics 2011;31:1741-55.

8. Nicolay S, De Foer B, Bernaerts A, Van Dinther J, Parizel PM. Aberrant internal carotid artery presenting as a retrotympanic vascular mass. Acta Radiol Short Rep 2014;26;3:2047981614553695.

9. Muderris T, Bercin S, Sevil E, Cetin H, Kiris M. A potentially catastrophic anatomical variation: aberrant internal carotid artery in the middle ear cavity. Case Rep Otolaryngol 2013;2013:743021.

10. Friedmann DR, Eubig J, Winata LS, Pramanik BK, Merchant SN, Lalwani AK. Prevalence of jugular bulb abnormalities and resultant inner ear dehiscence: a histopathologic and radiologic study. Otolaryngol Head Neck Surg 2012;147:750-6.

11. Shaikh MF, Mahboubi H, German M, Djalilian HR. A novel approach for surgical repair of dehiscent high jugular bulb. Laryngoscope 2013;123:1803-5. 
12. Koo YH, Lee JY, Lee JD, Hong HS. Dehiscent high-riding jugular bulb presenting as conductive hearing loss: a case report. Medicine (Baltimore) 2018;97:e11067.

13. Juliano AF, Ginat DT, Moonis G. Imaging review of the temporal bone: part II. Traumatic, postoperative, and noninflammatory nonneoplastic conditions. Radiology 2015;276:655-72.

ORCID ID:

G. Burakgazi 0000-0003-4913-4838; H. Bayaroğulları 0000-0001-6147-5876
14. Aralaşmak A, Dinçer E, Arslan G, Cevikol C, Karaali K. Posttraumatic labyrinthitis ossificans with perilymphatic fistulization. Diagn Interv Radiol 2009;15:239-41.

15. Gray BG, Willinsky RA, Rutka JA, Tator CH. Spontaneous meningocele, a rare middle ear mass AJNR Am J Neuroradiol 1995; $16: 203-7$.

\section{deomed.}

This is an open access article distributed under the terms of the Creative Commons Attribution-NonCommercial-NoDerivs 4.0 Unported (CC BY-NCND4.0) Licence (http://creativecommons.org/licenses/by-nc-nd/4.0/) which permits unrestricted noncommercial use, distribution, and reproduction in any medium, provided the original work is properly cited. How to cite this article: Burakgazi G, Bayaroğulları H. Non-inflammatory and non-neoplastic soft tissue lesions of the tympanic cavity. Anatomy 2020;14(3):157-164. 